\title{
Differential equations and Feynman integrals
}

\author{
A. V. Kotikov \\ Bogoliubov Laboratory of Theoretical Physics \\ Joint Institute for Nuclear Research \\ 141980 Dubna, Russia
}

\begin{abstract}
The role of differential equations in the process of calculating Feynman integrals is shown. An example of a diagram is given, in the calculation of which the method of differential equations was introduced, the properties of the inverse-mass-expansion coefficients are shown, and modern methods based on differential equations are considered.
\end{abstract}

\section{Introduction}

The calculation of the Feynman integrals (FIs) provides basic information both for the matrix elements of the experimentally studied processes and for the characteristics of the physical models themselves (their renormalization, critical behavior, etc.). When studying renormalization and critical behavior, it is usually sufficient to restrict oneself to the limit of massless particles at which the corresponding two-point FIs are fairly simple. However, starting at 2 or 3 loop levels, there is a need to use modern methods such as Integration by Parts (IBP) [1] and Gegenbauer's polynomial method [2] (see also the recent paper [3] and reviews in [4] and [5]). 1

Calculating massive FIs (i.e. FIs having propagators of massive particles) is a much more complex problem. Simple results (in the form of a product of $\Gamma$-functions) exist only for simple tadpoles (see eq. (10) below), and a massive one-loop loop is already given by a one-fold integral (see eq. (15) below).

It turned out, however, that massive FIs satisfy IBP procedures [1], which lead to relations between FIs equivalent to the original ones, but with different powers of the propagators included in them, including powers equal to zero. Diagrams containing propagators with degrees equal to zero are equivalent to simpler diagrams obtained by canceling these propagators (and reducing the points they join to one point).

Such relationships can be understood in two ways. First, considering them algebraically, one can understand them as connections between diagrams that are not independent and can be reduced to a certain set of independent diagrams, which are called masters (or master integrals) [7].

Second, propagators with powers greater than 1 can be considered as derivatives (with respect to the corresponding mass or external momentum) from the propagator with a degree of 1 . Thus, the relationships between the master integrals can be considered differential equations (DEs) for these masters (see an example in Section 2), containing inhomogeneous terms, including only simpler diagrams (i.e., obtained from the original diagrams by reducing

\footnotetext{
${ }^{1}$ Note that multi point massless FIs are as complex as massive FIs (see, for example, Ref. [6] about the relationship between 2-point massive FIs and 3-point massless FIs).
} 
some propagator). For these simpler diagrams, one can obtain (by applying the IBP procedure to them) similar DEs (see Appendix A) containing inhomogeneous terms, including only even simpler diagrams (i.e., obtained from simple diagrams by reducing some propagator). By repeating the original procedure several times, it is usually possible to obtain DEs containing inhomogeneous terms, including only tadpoles, which in turn are easily computable exactly (note, however, that starting from the 2-loop level, obtaining results for massive tadpoles requires the use of modern methods for FI calculating (see [8] and references and discussions therein)). 2 More complicated diagrams can be obtained from these tadpoles by solving successively obtained DEs with some boundary conditions. For (dimensionally regularized) massive FIs, a good boundary condition is the limit of infinitely large mass, at which these diagrams usually vanish.

In Section 2 we will consider a two-loop FI, the calculation of which leds to the use of differential equations. The calculation of massive diagrams is given in Section 3: rules are given for their efficient calculation, examples of two- and three-point diagrams are considered. The recurrence relations for the coefficients of decomposition in inverse mass are considered. In Section 4 a short review of modern computing technology is given. The appendix A contains the derivation of DEs for massive diagrams from the inhomogeneous term of the DE for the diagram considered in Section 2.

\section{History}

As mentioned in the introduction, integral representations for one-loop FIs (obtained, for example, using the Feynman parameter method [9]) are hypergeometric functions 3 and, thus, can be represented as solutions of some DEs. The importance of DEs for FIs was recognized long ago (see, for example, [11, 12]). However, in my opinion, the practical application awaited the emergence of IBP procedure [1] for FIs and is based on the use of IBP relations (see eqs. (16) and (17) below).

IBP-based DEs appeared in the nineties in several works, studying FIs: massive two-point ones in [13, 14], massive three-point ones in [13], four-point ones in [16] and $\hat{n}$-point ones [17, 18] (a short short overview made at that time can be found in the issue [19] dedicated to the 70th anniversary of Academician O.S. Parasyuk, co-author of the BPHZ renormalization procedure [20] (see, for example, the book [21]). 4]

In the case of Refs. [13, 25], the starting point was to study a preprint of the excellent yet unpublished work [7] on the calculation of two-loop massive FIs. Despite the excellent results, the paper itself (i.e. the conclusions of these results) turned out to be quite difficult to understand.

I decided to reproduce these results using the IBP relations, which proved to be very

\footnotetext{
${ }^{2}$ Sometimes it is convenient to stop the considered procedure on one-loop massive FI, the calculation of which can be performed, for example, by the method of Feynman parameters (see, for example, 9].

${ }^{3}$ Investigations of hypergeometric functions related to the calculation of FI are recently presented [10] as contribution to the Proceedings.

${ }^{4}$ The results for massless diagrams are sometimes useful to obtain in $x$-space (see [22, 23]). It is convenient to do this in $x$-space for so-called dual diagrams (see, for example, 23, 24]). A dual diagram is obtained from the initial one by replacement of all $p$ by $x$ with the rules of correspondence between the graph and the integral, as in a $x$-space. For massive two-pint and three-point diagrams the study in dual $x$-space done in Refs. 25] and [26, respectively.
} 
successful for calculating the correction to the longitudinal structure function of the deepinelastic scattering (DIS) [24, 27]. Indeed, the developed method [23, 24] for calculating massless FIs containing the (traceless) product of impulses in the numerators of propagators was based on the application of IBP to such diagrams. This method, extended to 3-, 4and 5-loop diagrams and built into computer programs, is the basis of modern calculations, starting with the excellent work in which NNLO corrections for anomalous dimensions of Wilson operators were obtained (see the recent paper [28] and references and discussions therein). A similar method has also been developed [29] to calculate massive corrections in the DIS process (see the recent papers [30, 31] and the review [32] and references and discussions therein).

The first example was studied in [13, 25] is the diagram

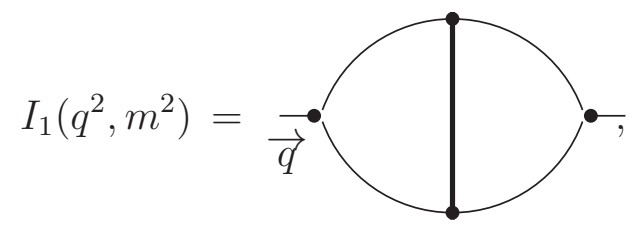

having the vertical massive propagator (see eq. (9) for definitions). The diagram has leftright and top-bottom symmetries.

Applying IBP relations (16) to the left triangle of the diagram $I_{1}\left(q^{2}, m^{2}\right)$ in succession with vertical and lateral distinguished lines, we get

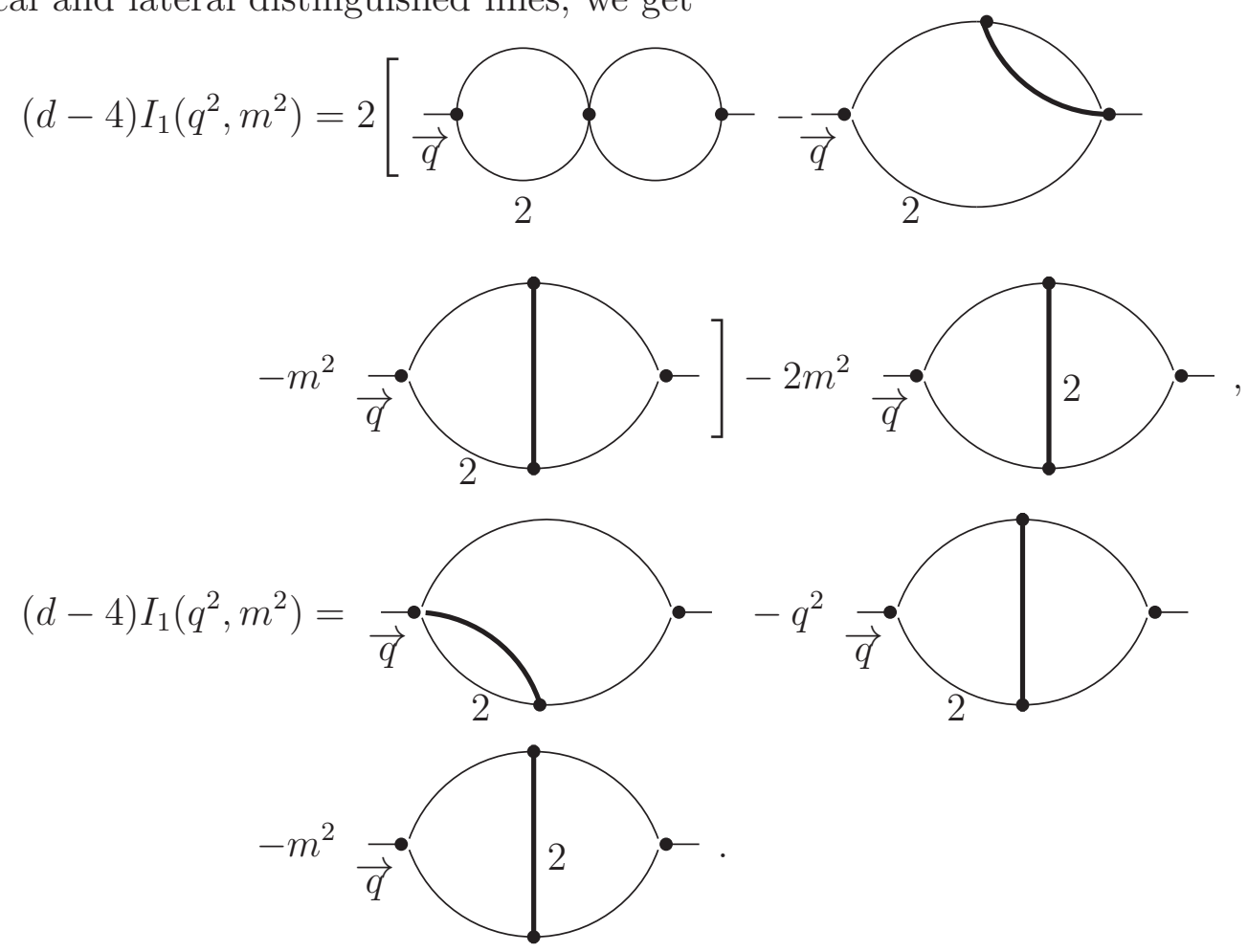

Taking the combination of these equations: Eq. (22) $-2\left(m^{2} / q^{2}\right) \times$ Eq.(3)), we have

$$
(d-4)\left(1-\frac{2 m^{2}}{q^{2}}\right) I_{1}\left(q^{2}, m^{2}\right)=2 J_{1}\left(q^{2}, m^{2}\right)-2 m^{2}\left(1-\frac{m^{2}}{q^{2}}\right) \rightarrow
$$


where

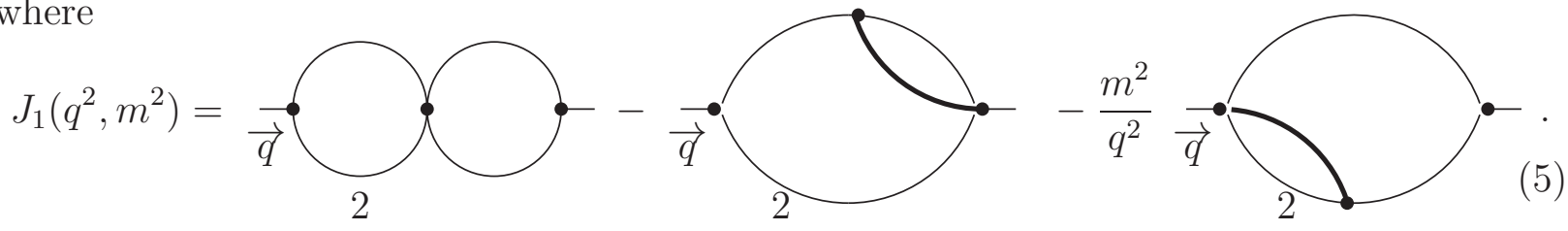

Because

$$
\frac{1}{\left(q^{2}+m^{2}\right)^{2}}=-\frac{d}{d m^{2}} \frac{1}{\left(q^{2}+m^{2}\right)^{2}}
$$

the Eq. (5) can be rewritten in the form

$$
\left[(d-4)\left(1-\frac{2 m^{2}}{q^{2}}\right)-2 m^{2}\left(1-\frac{m^{2}}{q^{2}}\right) \frac{d}{d m^{2}}\right] I_{1}\left(q^{2}, m^{2}\right)=2 J_{1}\left(q^{2}, m^{2}\right),
$$

i.e. the first order DE 5 for the original diagram with the inhomogeneous term $J_{1}\left(q^{2}, m^{2}\right)$ containing only simpler diagrams (i.e. obtained from the original by canceling one of the propagators) (see eq. (15)).

The first diagram in the inhomogeneous term $J_{1}\left(q^{2}, m^{2}\right)$ is independent of mass and therefore can be easily calculated as a product of the $\Gamma$-functions (see eq. (11) below):

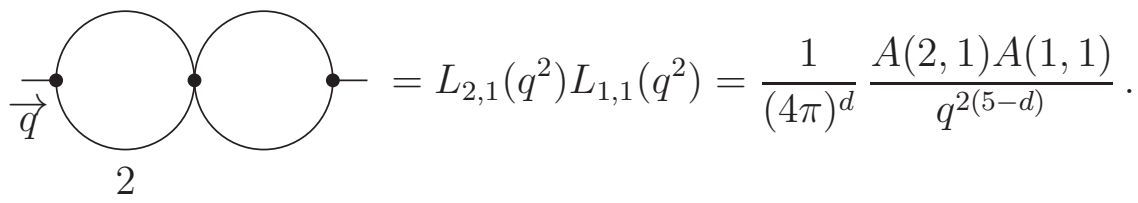

where $A\left(\alpha_{1}, \alpha_{2}\right)$ is given in eq. (13) below.

Using IBP relations, for the remaining two diagrams in the inhomogeneous $J_{1}\left(q^{2}, m^{2}\right)$ term diagrams, one can obtain similar equations with inhomogeneous terms containing only even simpler diagrams (i.e., obtained from the original by canceling two propagators). These results can be found in the Appendix A.

\section{Calculation of massive Feynman integrals}

Let us briefly consider the rules for calculating diagrams having the massive propagators.

1. Massless propagator and the propagator with mass $m$ will be represented as

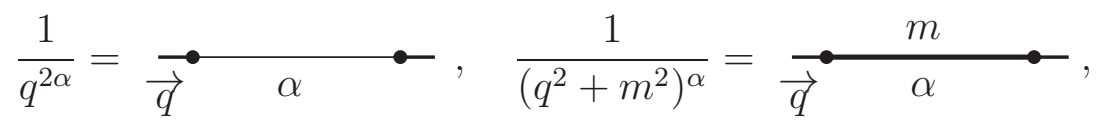

where the symbol $m$ will be omitted in the one-mass case (as in the case of $I_{1}\left(q^{2}, m^{2}\right)$ in eq. (1)).

\footnotetext{
${ }^{5}$ Hereafter we consider only the first order DE. The consideration of the high order DEs can be found in section 7 of the review 32 .
} 
2. The massive one-loop tadpole $T_{\alpha_{1}, \alpha_{2}}\left(m^{2}\right)$ and the massless loop $L_{\alpha_{1}, \alpha_{2}}\left(q^{2}\right)$ can be calculated exactly as combinations of the $\Gamma$-functions:

$$
\begin{aligned}
& T_{\alpha_{1}, \alpha_{2}}\left(m^{2}\right)=\int \frac{D k}{k^{2 \alpha_{1}}\left(k^{2}+m^{2}\right)^{\alpha_{2}}}=>\overbrace{}^{\alpha_{1}}=\frac{R\left(\alpha_{1}, \alpha_{2}\right)}{m^{2\left(\alpha_{1}+\alpha_{2}-d / 2\right)}}, \\
& L_{\alpha_{1}, \alpha_{2}}\left(q^{2}\right)=\int \frac{D k}{(q-k)^{2 \alpha_{1}} k^{2 \alpha_{2}}}=\underset{q}{\alpha_{2}}=\frac{A\left(\alpha_{1}, \alpha_{2}\right)}{q^{2\left(\alpha_{1}+\alpha_{2}-d / 2\right)}},
\end{aligned}
$$

where

$$
\begin{aligned}
& A\left(\alpha_{1}, \alpha_{2}\right)=\frac{a\left(\alpha_{1}\right) a\left(\alpha_{2}\right)}{a\left(\alpha_{1}+\alpha_{2}-d / 2\right)}, \quad a(\alpha)=\frac{\Gamma(\tilde{\alpha})}{\Gamma(\alpha)}, \quad \tilde{\alpha}=\frac{d}{2}-\alpha, \\
& R\left(\alpha_{1}, \alpha_{2}\right)=\frac{\Gamma\left(d / 2-\alpha_{1}\right) \Gamma\left(\alpha_{1}+\alpha_{2}-d / 2\right)}{\Gamma(d / 2) \Gamma\left(\alpha_{2}\right)}
\end{aligned}
$$

and

$$
D k=\frac{d^{d} k}{\pi^{d / 2}}=(4 \pi)^{d / 2} D_{E} k, \quad D_{E} k=\frac{d^{d} k}{(2 \pi)^{d}} .
$$

Here $D_{E} k$ is the usual Euclidean measure in in $(d=4-2 \varepsilon)$ space.

3. A simple loop of two massive propagators with masses $m_{1}$ and $m_{2}$ can be represented as hypergeometric function, which can be calculated in a general form, for example, by Feynman-parameter method (see [9]). It is very convenient, using this approach to represent the loop as an integral of a propagator with the "effective mass" $\mu$ [13, 33, 34, 35, 36, 37, 38]:

$$
\begin{aligned}
& \int \frac{D k}{\left[(q-k)^{2}+m_{1}^{2}\right]^{\alpha_{1}}\left[k^{2}+m_{2}^{2}\right]^{\alpha_{2}}}=\frac{\Gamma\left(\alpha_{1}+\alpha_{2}-d / 2\right)}{\Gamma\left(\alpha_{1}\right) \Gamma\left(\alpha_{2}\right)} \\
& \times \int_{0}^{1} \frac{d s s^{\alpha_{1}-1}(1-s)^{\alpha_{2}-1}}{\left[s(1-s) q^{2}+m_{1}^{2} s+m_{2}^{2}(1-s)\right]^{\alpha_{1}+\alpha_{2}-d / 2}}=\frac{\Gamma\left(\alpha_{1}+\alpha_{2}-d / 2\right)}{\Gamma\left(\alpha_{1}\right) \Gamma\left(\alpha_{2}\right)} \\
& \times \int_{0}^{1} \frac{d s}{s^{1-\tilde{\alpha}_{2}}(1-s)^{1-\tilde{\alpha}_{1}}} \frac{1}{\left[q^{2}+\mu^{2}\right]^{\alpha_{1}+\alpha_{2}-d / 2}}, \quad\left(\mu^{2}=\frac{m_{1}^{2}}{1-s}+\frac{m_{2}^{2}}{s}\right) .
\end{aligned}
$$

It is useful to rewrite the equation graphically as

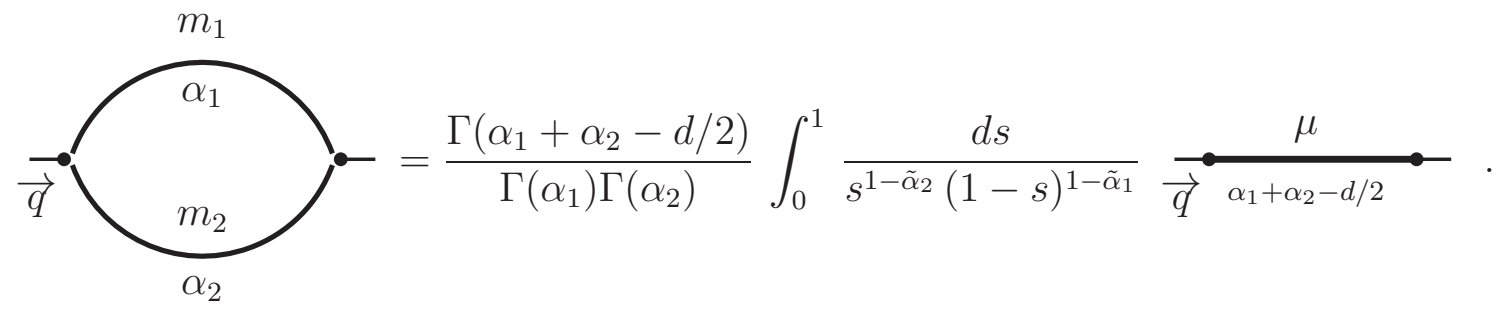

The rule is very convenient in the cases $m_{2}=0$ and $m_{1}=m_{2}$, where the variable $\mu$ is equal to $\mu^{2}=m_{1}^{2} / s$ and $\mu^{2}=m_{1}^{2} / s(1-s)$, respectively. Such simple forms of $\mu$ gives a 
possibility to use directly an inverse-mass expansion without a usage of Mellin-Barnes representation, which is essentially more complicated procedure.

4. For any triangle with indices $\alpha_{i}(i=1,2,3)$ and masses $m_{i}$ there is the following relation, which is based on integration by parts (IBP) procedure [1, 13, 15]

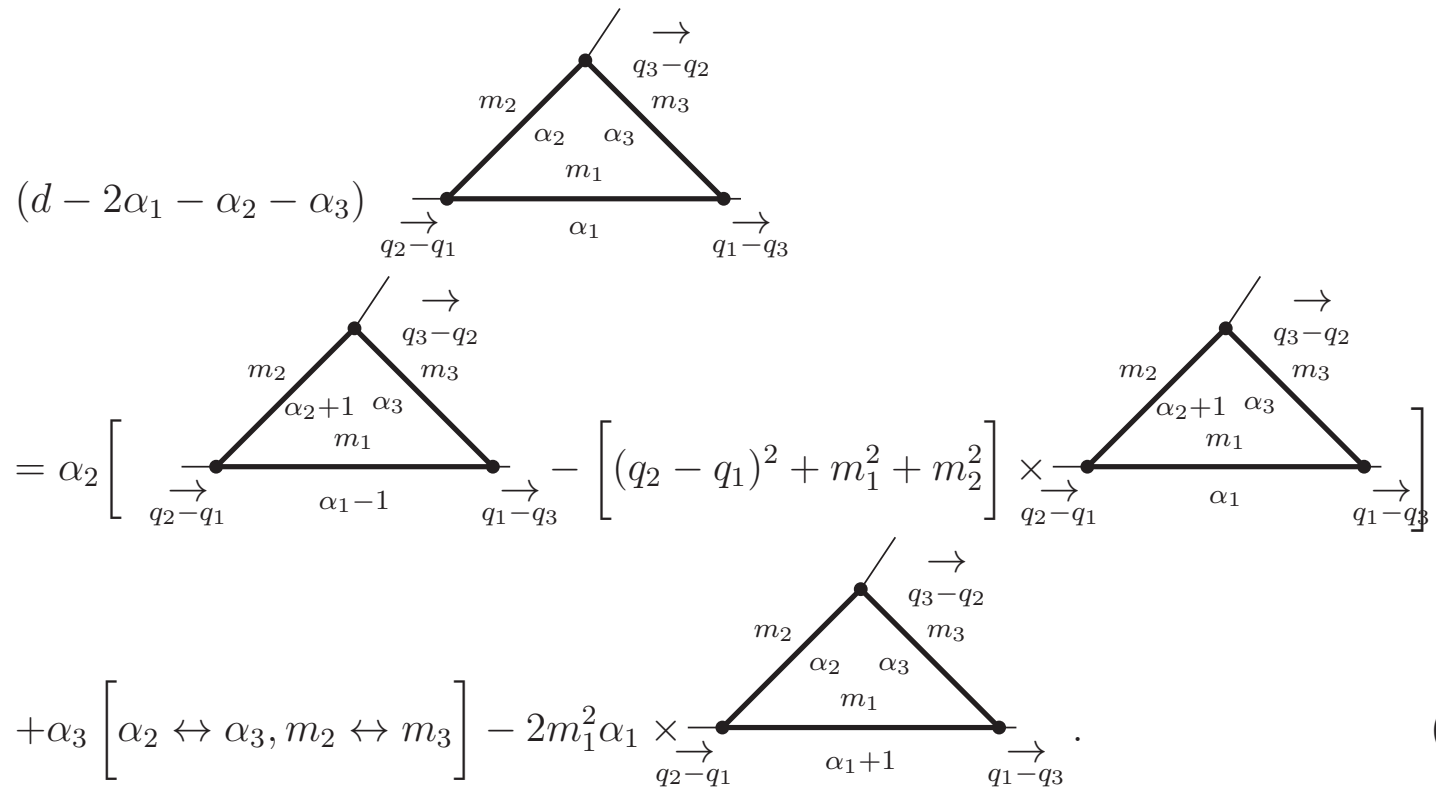

Eq. (16) can been obtained by introducing the factor $\left(\partial / \partial k_{\mu}\right)\left(k-q_{1}\right)^{\mu}$ to the subintegral expression of the triangle, shown below as [...], and using the integration by parts procedure as follows:

$$
\begin{aligned}
& d \int D k[\ldots]=\int D k\left(\frac{\partial}{\partial k_{\mu}}\left(k-q_{1}\right)^{\mu}\right)[\ldots]=\int D k \frac{\partial}{\partial k_{\mu}}\left(\left(k-q_{1}\right)^{\mu}[\ldots]\right) \\
& -\int D k\left(k-q_{1}\right)^{\mu} \frac{\partial}{\partial k_{\mu}}([\ldots])
\end{aligned}
$$

The first term in the r.h.s. becomes to be zero because it can be represented as a surface integral on the infinite surface. Evaluating the second term in the r.h.s. we reproduce Eq. (16). Note that the equation (17) can also be applied to the $\hat{n}$-point subgraph (see, for example, [17]).

As it is possible to see from Eqs. (16) and (17) the line with the index $\alpha_{1}$ is distinguished. The contributions of the other lines are same. So, we will call below the line with the index $\alpha_{1}$ as a "distinguished line". It is clear that a various choices of the distinguished line produce different types of the IBP relations.

\subsection{Basic massive two-loop integrals}

Below in the present analysis we will concentrate mostly on two-loop two-point and threepoint diagrams, which can be taken from the diagram shown in Fig. 1. We will call them 


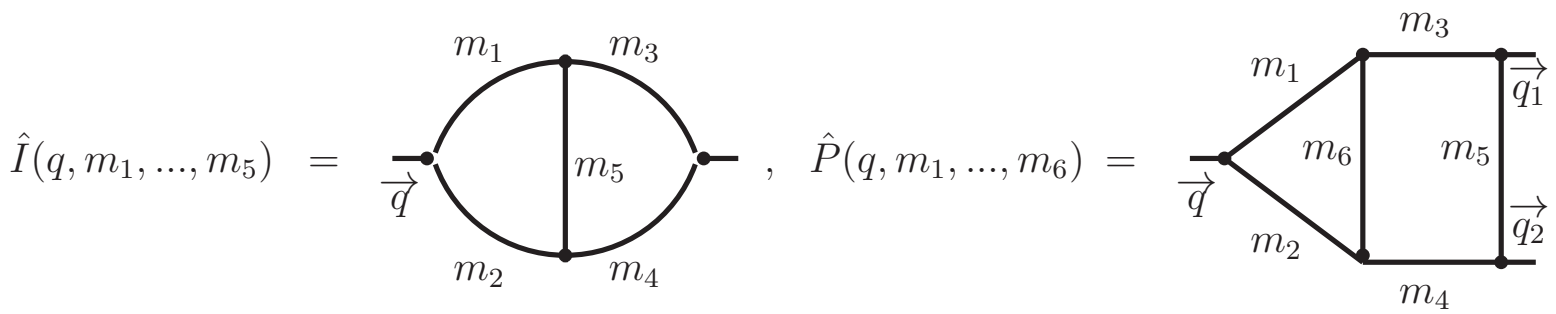

Figure 1: Two-loop two-point diagram $\hat{I}\left(q, m_{1}, \ldots, m_{5}\right)$ and three-point diagram $\hat{P}\left(q, m_{1}, \ldots, m_{6}\right)$ with $q_{1}^{2}=q_{2}^{2}=0$.

as:

$$
\begin{aligned}
& \hat{I}_{j}=\hat{I}\left(q, m_{j}=m \neq 0, m_{p}=0, p \neq j\right), \quad \hat{I}_{i j}=\hat{I}\left(q, m_{i}=m_{j}=m \neq 0, m_{p}=0, p \neq i \neq j\right), \\
& \hat{I}_{i j s}=\hat{I}\left(q, m_{i}=m_{j}=m_{s}=m \neq 0, m_{p}=0, p \neq i \neq j \neq s\right), \\
& \hat{I}_{i j s t}=\hat{I}\left(q, m_{i}=m_{j}=m_{s}=m_{t}=m \neq 0, M_{p}=0, p \neq i \neq j \neq s \neq t\right), \\
& \hat{P}_{j}=\hat{P}\left(q, m_{j}=m \neq 0, m_{p}=0, p \neq j\right), \quad \hat{P}_{i j}=\hat{P}\left(q, m_{i}=m_{j}=m \neq 0, m_{p}=0, p \neq i \neq j\right), \\
& \hat{P}_{i j s}=\hat{P}\left(q, m_{i}=m_{j}=m_{s}=m \neq 0, m_{p}=0, p \neq i \neq j \neq s\right), \\
& \hat{P}_{i j s t}=\hat{P}\left(q, m_{i}=m_{j}=m_{s}=m_{t}=m \neq 0, m_{p}=0, p \neq i \neq j \neq s \neq t\right) .
\end{aligned}
$$

Now we repeat once again the procedure of the DE method. Application of the IBP procedure [1] to loop internal momenta leads to relations between various FIs and, therefore, to the necessity of calculating only some of them, which in a sense are independent. These independent diagrams (which were chosen completely arbitrarily, of course) are called master integrals [7].

Applying the IBP procedure [1] to the master-integrals themselves leads to DEs [13, 25] for them with the inhomogeneous terms containing less complex diagrams. Applying the IBP procedure to diagrams in inhomogeneous terms leads to new DEs for them with new inhomogeneous terms containing even more less complex diagrams (三 less ${ }^{2}$ complex ones). By repeating the procedure several times, in the last step we can obtain inhomogeneous terms containing mainly tadpoles, which can be easily calculated in-turn.

Solving the corresponding DEs in this last step, diagrams for the inhomogeneous terms of the DEs in the previous step can be reproduced. Repeating the procedure several times, me can get the results for the original Feynman diagram.

Thus, the DE method procedure is well defined, but it requires a lot of manual work and a lot of time. So, the calculations [34] of each of the diagrams $P_{6}$ and $P_{126}$ took about a month of work (of course, along with checking the results). It would be nice, however, to transfer some of the work to the computer. The first attempt based on the properties of the inverse mass expansion coefficients of the master integrals is presented in the next Section. A more modern and efficient technique is discussed in Section 5.

\section{Evaluation of series}

Calculations of the two-point diagrams shown in Fig. 1, which do not contain elliptic structures (see Fig. 2 in Ref. [35]), 6 as well as calculations of some three-point diagrams shown

\footnotetext{
${ }^{6}$ In fact, the results for these two-point diagrams were found in the late eighties and early nineties, and were planned to be published in a long paper summarizing the results done in Refs. 13, 15]. However, this paper has not been published. These results, after verification, were published in Ref. [35].
} 
in Fig. 1 (see also Fig. 3 in Ref. [35]) lead to results with interesting properties of their inverse mass expansion coefficients.

\subsection{Properties of series}

The inverse-mass expansion of two-loop two-point and three-point diagrams 7 with one nonzero mass (massless and massive propagators are shown by dashed and solid lines, respectively), can be considered as

$$
\begin{aligned}
& \mathrm{FI}=\frac{\hat{N}}{q^{2 \alpha}} \sum_{n=1} C_{n}(\eta x)^{n}\left\{F_{0}(n)+\left[\ln x F_{1,1}(n)+\frac{1}{\varepsilon} F_{1,2}(n)\right]\right. \\
& \left.+\left[\ln ^{2} x F_{2,1}(n)+\frac{1}{\varepsilon} \ln x F_{2,2}(n)+\frac{1}{\varepsilon^{2}} F_{2,3}(n)+\zeta(2) F_{2,4}(n)\right]+\cdots\right\},
\end{aligned}
$$

where $x=q^{2} / m^{2}, \eta=1$ or -1 and $\alpha=1$ and 2 for two-point and three-point cases, respectively. The normalization $\hat{N}=\left(\bar{\mu}^{2} / m^{2}\right)^{2 \varepsilon}$, where $\bar{\mu}=4 \pi e^{-\gamma_{E}} \mu$ is in the standard $\overline{M S}$-scheme and $\gamma_{E}$ is the Euler constant. Moreover,

$$
C_{n}=\frac{(n !)^{2}}{(2 n) !} \equiv \hat{C}_{n}
$$

for diagrams with two-massive-particle-cuts (2m-cuts). For the diagrams with one-massiveparticle-cuts ( $m$-cuts) $C_{n}=1$.

For $m$-cut case, the coefficients $F_{N, k}(n)$ should have the form

$$
F_{N, k}(n) \sim \frac{S_{ \pm a, \ldots}}{n^{b}}, \frac{\zeta( \pm a)}{n^{b}}
$$

where $S_{ \pm a, \ldots} \equiv S_{ \pm a, \ldots}(j-1)$ are nested sums [39]: 8

$$
S_{ \pm a}(j)=\sum_{m=1}^{j} \frac{(-1)^{m}}{m^{a}}, \quad S_{ \pm a, \pm b, \ldots}(j)=\sum_{m=1}^{j} \frac{(-1)^{m}}{m^{a}} S_{ \pm b, \ldots}(m),
$$

and $\zeta( \pm a)=S_{ \pm a}(\infty)$ and $\zeta( \pm a,, \pm b, \ldots)=S_{ \pm a, \pm b, \ldots}(\infty)$ are the Euler-Zagier constants.

For $2 m$-cut case, the coefficients $F_{N, k}(n)$ can be more complicated

$$
F_{N, k}(n) \sim \frac{S_{ \pm a, \ldots}}{n^{b}}, \frac{V_{a, \ldots}}{n^{b}}, \frac{W_{a, \ldots}}{n^{b}},
$$

where $W_{ \pm a, \ldots} \equiv W_{ \pm a, \ldots}(j-1)$ and $V_{ \pm a, \ldots} \equiv V_{ \pm a, \ldots}(j-1)$ with 35]

$$
\begin{aligned}
& W_{a}(j)=\sum_{m=1}^{j} \frac{\hat{C}_{m}^{-1}}{m^{a}}, \quad W_{a, b, c, \cdots}(j)=\sum_{m=1}^{j} \frac{\hat{C}_{m}^{-1}}{m^{a}} S_{b, c, \cdots}(m), \\
& V_{a}(j)=\sum_{m=1}^{j} \frac{\hat{C}_{m}}{m^{a}}, \quad V_{a, b, c, \cdots}(j)=\sum_{m=1}^{j} \frac{\hat{C}_{m}}{m^{a}} S_{b, c, \cdots}(m),
\end{aligned}
$$

\footnotetext{
${ }^{7}$ The diagrams are complicated two-loop FIs that do not have cuts of three massive particles. thus, their results should be expressed as combinations of Polylogarithms. Note that we consider only three-point diagrams with independent upward momenta $q_{1}$ and $q_{2}$, which satisfy the conditions $q_{1}^{2}=q_{2}^{2}=0$ and $\left(q_{1}+q_{2}\right)^{2} \equiv q^{2} \neq 0$, where $q$ is downward momentum.

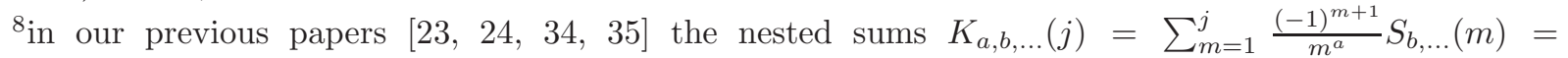
$-S_{-a, b, \ldots}(j)$ have been used together with their analytic continuations [24, 40].
} 
The terms $\sim V_{a, \ldots}$ and $\sim W_{a, \ldots}$ can come only in the $2 m$-cut case. The origin of the appearance of these terms is the product of series (20) with the different coefficients $C_{n}=1$ and $C_{n}=\hat{C}_{n}$.

\subsection{Two-point examples}

As an example, consider two-loop two-point diagrams $\hat{I}_{5}$ and $\hat{I}_{12}$ studied in 35

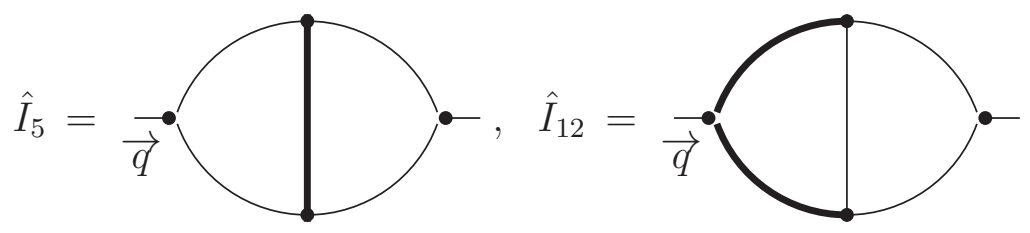

where $\hat{I}_{5}$ coincides with $I_{1}\left(q^{2}, m^{2}\right)$ considered in Section 2 .

Their results are

$$
\begin{aligned}
& \hat{I}_{5}=\frac{\hat{N}}{q^{2}} \sum_{n=1} \frac{x^{n}}{n}\left\{\ln ^{2} x-\frac{2}{n} \ln x+2 \zeta(2)+4 S_{-2}+2 \frac{2}{n^{2}}+\frac{2(-)^{n}}{n^{2}}\right\} \\
& \hat{I}_{12}=-\frac{\hat{N}}{q^{2}} \sum_{n=1} \frac{(-x)^{n}}{n^{2}}\left\{\frac{1}{n}+\hat{C}_{n}\left(-2 \ln x-3 W_{1}+\frac{2}{n}\right)\right\} .
\end{aligned}
$$

From (28) one can see that the corresponding functions $F_{N, k}(n)$ have the form

$$
F_{N, k}(n) \sim \frac{1}{n^{3-N}}, \quad(N \geq 2)
$$

if we introduce the following complexity of the sums $(\bar{\Phi}=(S, V, W))$

$$
\bar{\Phi}_{ \pm a} \sim \bar{\Phi}_{ \pm a_{1}, \pm a_{2}} \sim \bar{\Phi}_{ \pm a_{1}, \pm a_{2}, \cdots, \pm a_{m}} \sim \zeta_{a} \sim \frac{1}{n^{a}}, \quad\left(\sum_{i=1}^{m} a_{i}=a\right)
$$

The number $3-N$ determines the level of transcendentality (or complexity, or weight) of the coefficients $F_{N, k}(n)$. The property greatly reduces the number of the possible elements in $F_{N, k}(n)$. The level of transcendentality decreases if we consider the singular parts of diagrams and/or coefficients in front of $\zeta$-functions and of logarithm powers. Thus, finding the parts we can predict the rest using the ansatz based on the results already obtained, but containing elements with a higher level of transcendentality.

Other two-loop two-point integrals in 35] have similar form. They were exactly calculated by DE method [13, 25. Their representations in the form of Nielsen Polylogarithms [41] can be found also in Ref. [35].

\subsection{Three-point examples}

Now we consider two-loop three-point diagrams, $\hat{P}_{5}$ and $\hat{P}_{12}$ :

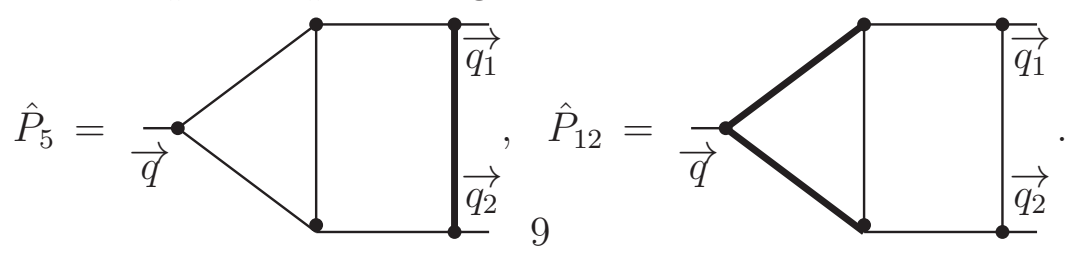


Their results are (see 35]):

$$
\begin{aligned}
\hat{P}_{5}= & \frac{\hat{N}}{\left(q^{2}\right)^{2}} \sum_{n=1} \frac{x^{n}}{n}\left\{-6 \zeta_{3}+2 S_{1} \zeta_{2}+6 S_{3}-2 S_{1} S_{2}+4 \frac{S_{2}}{n}-\frac{S_{1}^{2}}{n}+2 \frac{S_{1}}{n^{2}}\right. \\
& \left.+\left(-4 S_{2}+S_{1}^{2}-2 \frac{S_{1}}{n}\right) \ln x+S_{1} \ln ^{2} x\right\} \\
\hat{P}_{12}= & \frac{\hat{N}}{\left(q^{2}\right)^{2}} \sum_{n=1} \frac{(-x)^{n}}{n^{2}} \hat{C}_{n}\left\{\frac{2}{\varepsilon^{2}}+\frac{2}{\varepsilon}\left(S_{1}-3 W_{1}+\frac{1}{n}-\ln x\right)-6 W_{2}-18 W_{1,1}\right. \\
& \left.-13 S_{2}+S_{1}^{2}-6 S_{1} W_{1}+2 \frac{S_{1}}{n}+\frac{2}{n^{2}}-2\left(S_{1}+\frac{1}{n}\right) \ln x+\ln ^{2} x\right\},
\end{aligned}
$$

Now the coefficients $F_{N, k}(n)$ have the form

$$
F_{N, k}(n) \sim \frac{1}{n^{4-N}}, \quad(N \geq 3)
$$

The diagram $P_{5}$ (and also $P_{1}, P_{3}, P_{6}$ and $P_{126}$ in [35]) was calculated exactly by differential equation method [13, 25, 9 To find the results for $P_{12}$ (and also all others in [35]) we have used the knowledge of the several $n$ terms in the inverse-mass expansion (20) (usually less than $n=100$ ) and the following arguments:

- If a two-loop two-point diagram with a "similar topology" (for example, $I_{12}$ for $P_{12}$, etc.) was already calculated, we should consider a similar set of basic elements for corresponding $F_{N, k}(n)$ of two-loop three-point diagrams but with a higher level of complexity.

- Let the diagram under consideration contain singularities and/or powers of logarithms. Since the coefficients are very simple before the leading singularity, or the largest degree of the logarithm, or the largest $\zeta$-function, they can often be predicted directly from the first few terms of the expansion.

Moreover, often we can calculate the singular part using a different technique (see [35] for extraction of $\sim W_{1}(n)$ part). Then we should expand the singular parts, find the main elements and try to use them (with the corresponding increase in the level of complexity) in order to predict the regular part of the diagram. If we need to find $\varepsilon$-suppressed terms, we should increase the level of complexity of the corresponding basic elements.

Later, using the ansatz for $F_{N, k}(n)$ and several terms (usually less than 100) in the above expression, which can be exactly calculated, we obtain a system of algebraic equations for the parameters of the ansatz. Solving the system, we can obtain the analytical results for FIs without exact calculations. To check the results, we only need to calculate a few more terms in the above inverse-mass expansion (20) and compare them with the predictions of our anzatz with the fixed coefficients indicated above.

Thus, the considered arguments give a possibility to find results for many complicated two-loop three-point diagrams without direct calculations. Several process options have been

\footnotetext{
${ }^{9}$ The evaluation of inverse mass expansion coefficients is demonstrated in Ref. [36].
} 
successfully used to calculate Feynman diagrams for many processes (see [34, 35, 36, 37, 38, 42]).

Note that properties similar to (30) and (34) but $b=0$ in (22) was found for the eigenvalues of anomalous dimensions [43] and coefficient functions [44, as well as in the nextto-leading corrections [45] to the BFKL equation [46] for $N=4$ Super Yang-Mils (SYM) model. Such a strong restriction made it possible to obtain anomalous dimensions in the first three orders of the perturbation theory directly from the corresponding results for QCD (the "most complicated" parts are the same in $N=4$ SYM and QCD) [47, 48], as well as in the 4th, 5th, 6th and 7th orders (see [49, [50, 51] and 52, respectively) in the algebraic Bethe ansatz [53].

Note that the series (28), (29) and (32) can be expressed as a combination of the Nilson [41] and Remiddi-Vermaseren [54] polylogarithms with weight $4-N$ (see [35, 34]). More complicated cases were examined in [55].

\subsection{Properties of massive diagrams}

Coefficients of the inverse-mass-series expansions of the two-point and three-point FIs have the structure (30) and (34) with the rule (31). Note that these conditions greatly reduce the number of possible harmonic sums. In turn, the restriction is associated with a DE specific form for the considered FIs. The DEs can be formally represented as [56, 57] (see the example $I_{1}\left(q^{2}, m^{2}\right)$ considered in section 2$)$

$$
\left((x+a) \frac{d}{d x}-\bar{k}(x) \varepsilon\right) \mathrm{FI}=\text { less complicated } \operatorname{diagrams}\left(\equiv \mathrm{FI}_{1}\right),
$$

with some number $a$ and some function $\bar{k}(x)$. This form is generated by IBP procedure for diagrams including an inner $\hat{n}$-leg one-loop subgraph, which in turn contains the product $k^{\mu_{1}} \ldots k^{\mu_{m}}$ of its internal momenta $k$ with $m=\hat{n}-3$.

Indeed, for ordinary degrees $\alpha_{i}=1+a_{i} \varepsilon$ with arbitrary $a_{i}$ of subgraph propagators, the IBP relation (16) gives the coefficient $d-2 \alpha_{1}-\sum_{i=2}^{p} \alpha_{i}+m \sim \varepsilon$ for $m=n-3$. Important examples of applying the rule are the diagrams $\hat{I}_{5}, \hat{I}_{12}$ and $\hat{P}_{5}, \hat{P}_{12}$ (for the case $\hat{n}=2$ and $\hat{n}=3$ ) and also the diagrams in Ref. [58] (for the case $\hat{n}=3$ and $\hat{n}=4$ ). However, we note that the results for the non-planar diagrams (see Fig. 3 of [35]) obey the Eq. (34) but their subgraphs do not comply with the above rule. The disagreements may be related to the on-shall vertex of the subgraph, but this requires additional research.

Taking the set of less complicated Feynman integrals $\mathrm{FI}_{1}$ as diagrams having internal $\hat{n}$-leg subgraphs, we get their result structure similar to the one given above (34), but with a lower level of complexity. A)

So, the integrals $\mathrm{FI}_{1}$ should obey to the following equation (see $J_{2}^{(1)}\left(q^{2}, m^{2}\right)$ in Appendix

$$
\left(\left(x+a_{1}\right) \frac{d}{d x}-\bar{k}_{1}(x) \varepsilon\right) \quad \mathrm{FI}_{1}=\operatorname{less}^{2} \operatorname{complicated} \operatorname{diagrams}\left(\equiv \mathrm{FI}_{2}\right) .
$$

Thus, we will have the set of equations for all Feynman integrals $\mathrm{FI}_{\mathrm{n}}$ as

$$
\left(\left(x+a_{n}\right) \frac{d}{d x}-\bar{k}_{n}(x) \varepsilon\right) \quad \mathrm{FI}_{n}=\operatorname{less}^{n+1} \operatorname{complicated} \operatorname{diagrams}\left(\equiv \mathrm{FI}_{\mathrm{n}+1}\right)
$$


with the last integral $\mathrm{FI}_{\mathrm{n}+1}$ contains only tadpoles. Note that for the case $n=2$ the diagrams corresponding for the example $I_{1}\left(q^{2}, m^{2}\right)$, satisfy the system of equations, formally represented as eq. (37).

\section{Modern technique of massive diagrams}

In the last decade, several popular applications of DEs have emerged, allowing the use of computer resources and, thus, to obtain results for very complicated FIs.

In my opinion, the most successfully used are the so-called the canonical form [59] of DEs (and its generalizations in Refs. [60, 61]), the method [62] of simplified DEs, and the ability to use the effective mass (see eq. (15)), as well as their combinations. DEs are also effectively used in calculating FIs with an elliptical structure (see [63]).

\subsection{Canonical form of differential equations}

In our notation (see eqs. (35) - (37)), the canonical form [59], which was introduced by Johannes Henn in 2013 and and wildly popular now (a huge number of publications, which simply cannot be listed), represents a homogeneous matrix equation of the form (see also the review 64])

$$
\frac{d}{d x} \widehat{F I}-\varepsilon \widehat{K}(x) \widehat{F I}=0
$$

for the vector

$$
\widehat{F I}=\left(\begin{array}{l}
\mathrm{FI} \\
\mathrm{FI}_{1} / \varepsilon \\
\cdots \\
\mathrm{FI}_{\mathrm{n}} / \varepsilon^{\mathrm{n}}
\end{array}\right)
$$

where the matrix $\widehat{K}$ contains the functions $\bar{k}_{j} /\left(x+a_{j}\right)$ as its elements. The form (38) is called as the "canonic basic".

Note that obtaining it is far from trivial (see, for example, Appendix A for $\mathrm{FI}_{\mathrm{n}=2}$ diagrams). Moreover, it is not always achievable (see [60, 61]), where FIs were considered that are not reducible to (38) $)$, and its obtaining is sometimes associated with a nontrivial analysis (see Refs. 65] and [66] containing methods and criterion to obtain the equation, respectively). However, the form of (38) is very convenient as it can be easily diagonalized. Note that formally for real calculations of $\mathrm{FI}_{\mathrm{n}}$ it is convenient to replace

$$
\mathrm{FI}_{\mathrm{n}}=\widetilde{\mathrm{FI}}_{\mathrm{n}} \overline{\mathrm{FI}}_{\mathrm{n}}
$$

where the term $\overline{\mathrm{FI}}_{n}$ obeys the corresponding homogeneous equation

$$
\left(\left(x+a_{n}\right) \frac{d}{d x}-\bar{k}_{n}(x) \varepsilon\right) \overline{\mathrm{FI}}_{n}=0,
$$

The replacement simplifies the above equation (37) to the following form

$$
\left(x+a_{n}\right) \frac{d}{d x} \widetilde{\mathrm{FI}}_{n}=\widetilde{\mathrm{FI}}_{n+1} \frac{\overline{\mathrm{FI}}_{n+1}}{\overline{\mathrm{FI}}_{n}},
$$


having the solution

$$
\widetilde{\mathrm{FI}}_{n}(x)=\int_{0}^{x} \frac{d x_{1}}{x_{1}+a_{n}} \widetilde{\mathrm{FI}}_{n+1}\left(x_{1}\right) \frac{\overline{\mathrm{FI}}_{n+1}\left(x_{1}\right)}{\overline{\mathrm{FI}}_{n}\left(x_{1}\right)}
$$

Usually there are some cancellations in the ratio $\overline{\mathrm{FI}}_{n+1} / \overline{\mathrm{FI}}_{n}$ and sometimes it is equal to 1. In the last case, the equation (41) coincides with the definition of Goncharov Polylogariths [67] (see also the review [68] and the references therein).

Sometimes the integrand in (41) can have a quadratic form in the denominator, for example, $x_{1}^{2} \pm x_{1}+1$ ( $\operatorname{sign} \pm$ can change, including when passing from the Euclidean metric to the Minkowski metric). Such forms appeared in two-point FIs, $\hat{I}_{14}, \hat{I}_{15}$ and $\hat{I}_{123}$ and can be represented as Nilson three-logarithm with complicated argument, i.e. $\operatorname{Li}_{3}\left(-\mathrm{y}^{3}\right)$, where $y=(\sqrt{x+4}-x) /(\sqrt{x+4}+x)$ is so-called conformal variable, as well as in the transform in [69] of $H(-r, \ldots)$ functions, introduced in [70], to the Remiddi-Vermaseren polylopagitms [54] of variable $\sim y$ where one integral representation contains the factor $x_{1}^{2} \pm x_{1}+1$ in the denominator and is thus left in this form. We note that such terms come also in contributions of the massive form factors at 3-loop order [71. Later, the study of such integral representations leads to the discovery of cyclotomic Polylogarithms (see [72] and Ref. [32] for a review).

\subsection{Other approaches}

Here we will consider other methods that can be connected both with each other and with the canonical form and its generalizations (unfortunately, we cannot pretend here to be complete in listing all the approaches).

1. The simplified DE approach [62] is based on violation of momentum conservation by the parameter $x$, with some propagator. Using the IBP relations, we can obtain set of equations which depend on $x$. We can solve it with the boundary conditions at $x=0$ and take the limit $x \rightarrow 1$. The equations in this approach are usually representable in canonical form, which leads to very important results (see [73]).

2. Series expansions in singular and regular fixed points [74] (see also Ref. [75] and discussion therein) for DE systems, which generate eq. (38), for example, as

$$
\varepsilon \widehat{K}(x) \rightarrow \widehat{K}_{1}(x)+\varepsilon \widehat{K}_{2}(x) .
$$

The results are obtained in the form of Goncharov Polylogarithms [67] and, in some complicated cases, numerically.

3. Symmetries of FIs is a general method introduced in [76] which associates with any given Feynman diagram a system of partial DEs. The method uses the same variations which are used in the DE method [13] and IBP technique [1], but distinguishes itself by associating with any diagram a natural Lie group which acts on the diagram's parameter space. This approach was further developed and numerous diagrams have been analyzed within it (see the recent paper [77] and discussions and references therein).

4. Using the effective mass (15) reduces the number of loops in the considered diagram. In the cases under consideration, two-loop diagrams were reduced to one-loop ones. Then, one-loop diagrams were easily calculated using the DE method, and the required two-loop diagrams were presented as integrals of the obtained one-loop results (see Ref. [37]). 


\subsection{Elliptic structure}

Recently, the scientific community has centered its attention to the study of FIs whose geometric properties are defined by elliptic curves. We already have a lot of progress in understanding simplest functions beyond usual Polylogarithms, the so-called elliptic Polylogarithms (see the recent papers [63, 78, 79, 80] and references and discussions therein). Unfortunately, this topic is beyond the scope of this consideration (discussions about elliptic Polylogarithms can be found in Ref. [63], which is a contribution to the Proceedings), but we would like to point out only some of the integral representations that can be used in conjunction with elliptic Polylogarithms or even instead of elliptic Polylogarithms.

The effective mass form (15) turned out to be convenient for integrals containing an elliptic structure, since it allows one to represent the final result (see Ref. [37]) as an integral containing an elliptic kernel (i.e., a root of a polynomial of the 3rd or 4th degree) and a remainder represented in the form of an ordinary (Goncharov) polylogarithms. This approach can be an alternative to the introduction of elliptic Polylogarithms, which have a very complex structure (see, for example, the recent paper [81], where the study of sunsets in special kinematics was carried out both in the form of elliptic Polylogarithms (following Ref. [82]), as well as in the form of integral representations containing an elliptic kernel and ordinary Polylogarithms. Notice, that such analysis has been done in all orders of the dimensional regulator following the corresponding results in Ref. [83]).

At the end of the section, we would like to note about the recent paper [84], where the results for the most complex two-point single-mass diagrams containing an elliptical structure were obtained in the following form: using the effective mass representation, the original FIs were presented as integrals of one-loop diagrams dependent on the ratio $\mu / \mathrm{m}$. These one-loop diagrams were considered in a generalized canonical form (42). The authors of Ref. [84] have obtained very convenient representations for extremely complicated FIs.

\section{Conclusion}

In this short review, we examined the DE applicability for calculating FIs. We have considered an example $I_{1}\left(q^{2}, m^{2}\right)$, which led to the DE method sometime ago. The consistent application of IBP relations to $I_{1}\left(q^{2}, m^{2}\right)$, and then to the diagrams of inhomogeneous terms that arise each time, made it possible to obtain a DE hierarchy for increasingly simple diagrams obtained at each step by reducing one propagator. As noted in section 3.1, the DE method procedure is well defined but requires a lot of manual work and a lot of time.

Next, we showed an effective method restoring the exact result for two-point and threepoint two-loop diagrams in terms of inverse-mass-expansion coefficients, which have a beautiful structure and can be predicted using the corresponding coefficients at the poles or at transcendental constants such as Euler's $\zeta$-functions. These predictions were verified by analytical calculations of the first few terms using computer programs. Thus, this method is, apparently, the first, where computer programs were used for FI calculations using differential equations.

We have also given a brief overview of modern popular techniques such as the 'canonical form of DEs [59], the simplified DE approach [62] and the method of the effective mass (see, for example, Ref. [38]). Section 5.2 lists other popular approaches as well. 
The canonical form [59] (and its generalizations [60, 61]) are probably the most commonly used approaches (at least as a part of the calculations).

The effective mass method (see [38]) allows you to actually work with diagrams that have fewer loops than the original ones. The results for the original diagrams are obtained in the form of integral representations, where the integrand expressions are determined by calculating the diagrams with fewer loops. So, in Ref. [37] the two-loop diagrams with an elliptic structure were considered. The corresponding one-loop diagrams depending on the effective mass do not have an elliptical structure. Thus, the results of the original diagrams were presented in the form of integral representations containing an elliptic kernel (i.e., a root of a polynomial of the 3rd or 4th degree) and ordinary Polylogarithms. These representations can be used instead of elliptic Polylogarithms, and even more complex objects than elliptic Polylogarithms (see [84] and discussions therein).

Following the discussion in Section 5.3, the combined application of the effective-mass approach and generalizations of the canonical form for effective-mass-dependent diagrams can yield results for very complicated FIs. Such an analysis has already been carried out in the recent article [84] and, in our opinion, similar calculations can be performed in the near future for many complicated FIs.

Author thanks Johannes Blumlein for invitation to present a contribution to the proceedings of International Conference "Antidifferentiation and the Calculation of Feynman Amplitudes" (4-9 October 2020, Zeuthen, Germany) and Andrey Pikelner for help with axodraw2.

\section{Appendix A. Massive part of $J_{1}\left(q^{2}, m^{2}\right)$ in eq. (5).}

Now consider the following diagrams

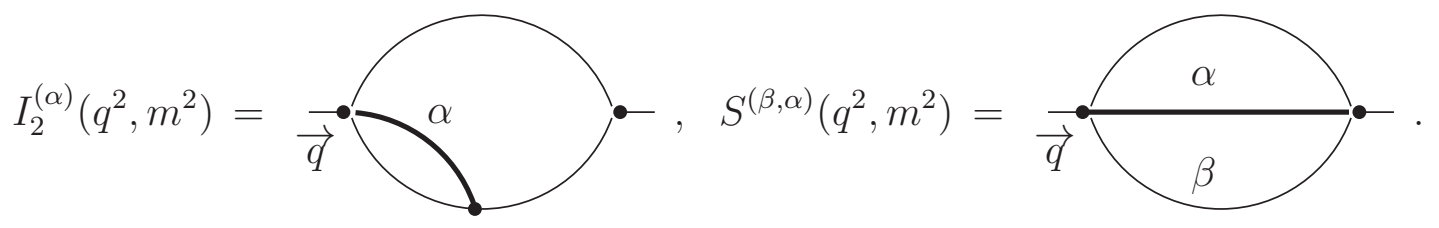

The IBP relations for the internal loop of the diagram produce two equations:

$$
\begin{aligned}
& (d-1-2 \alpha) I_{2}^{(\alpha)}\left(q^{2}, m^{2}\right)=\alpha J_{2}^{(\alpha+1)}\left(q^{2}, m^{2}\right)-m^{2} \alpha I_{2}^{(\alpha+1)}\left(q^{2}, m^{2}\right), \\
& (d-3) I_{2}^{(1)}\left(q^{2}, m^{2}\right)=T_{0,2}\left(m^{2}=0\right) L_{1,1}\left(q^{2}\right)-S^{(2,1)}\left(q^{2}, m^{2}\right)
\end{aligned}
$$

where

$$
J_{2}^{(\alpha)}\left(q^{2}, m^{2}\right)=T_{0, \alpha}\left(m^{2}\right) L_{1,1}\left(q^{2}\right)-S^{(1,2)}\left(q^{2}, m^{2}\right)
$$


We note that $T_{0,2}\left(m^{2}=0\right)=0$ in dimensional regularization and

$$
T_{0,2}\left(m^{2}\right) L_{1,1}\left(q^{2}\right)=\frac{1}{(4 \pi)^{d}} \frac{R(0,2) A(1,1)}{m^{2(2-d / 2)} q^{2(2-d / 2)}},
$$

where $R\left(\alpha_{1}, \alpha_{2}\right)$ and $A\left(\alpha_{1}, \alpha_{2}\right)$ are given in eqs. (13) and (12), respectively.

The IBP relations for internal triangles of the diagram $I_{2}^{(1)}\left(q^{2}, m^{2}\right)$ produce two additional equations:

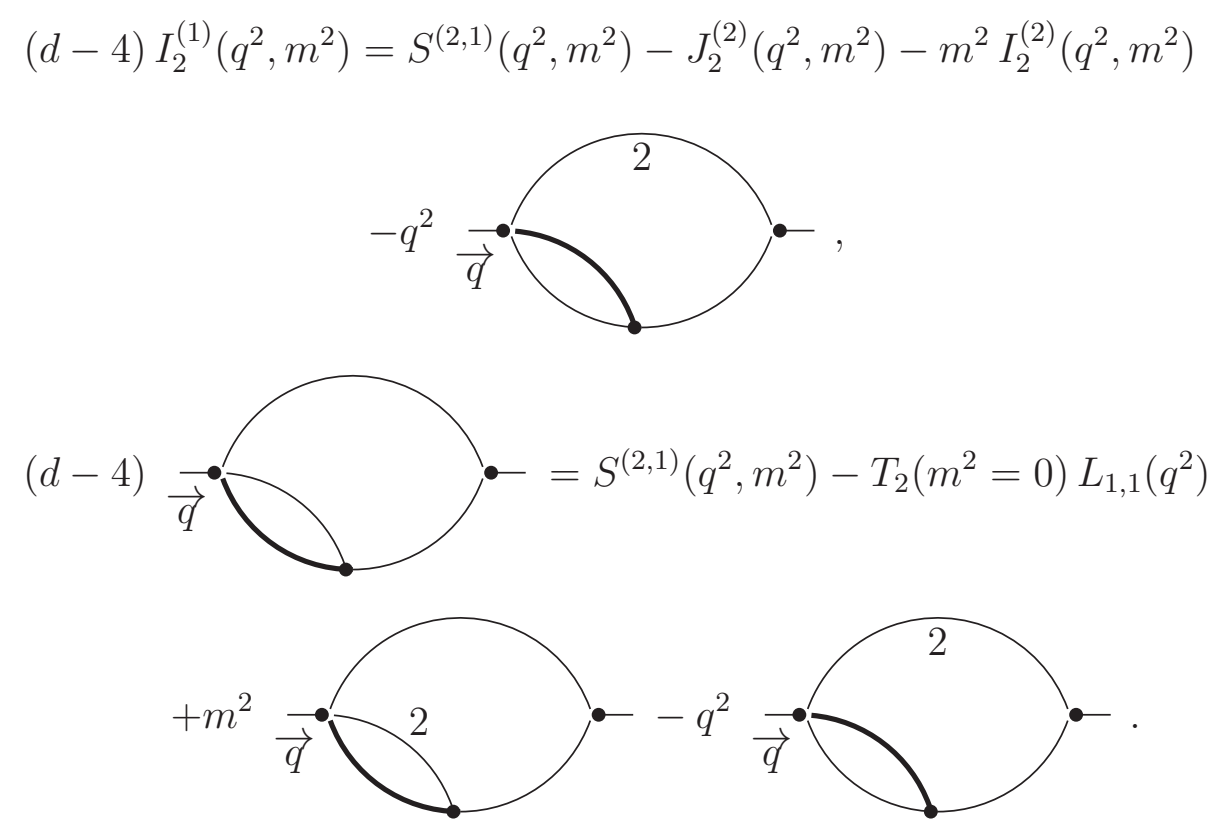

Using eqs. (A33) and (A7) as the combination: $2 \times(\underline{\mathrm{A} 3})+(\underline{\mathrm{A}} \mathrm{A})$, we have

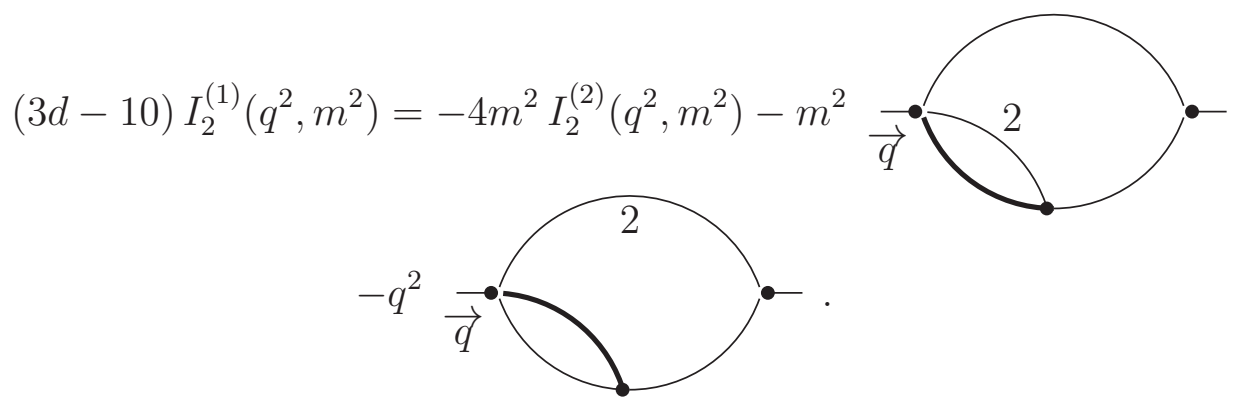

So, we have for the mass-dependent part of $J_{1}\left(q^{2}, m^{2}\right)$ (see eq. (15))

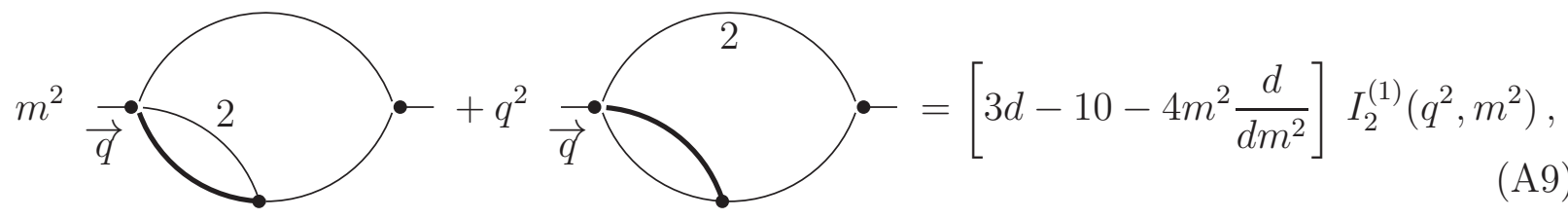

i.e. the mass-dependent combinations is expressed through the diagram $I_{2}^{(1)}\left(q^{2}, m^{2}\right)$ and its derivative. 
Using eq. (A2),

$$
\left[d-2-\alpha-m^{2} \frac{d}{d m^{2}}\right] I_{2}^{(\alpha)}\left(q^{2}, m^{2}\right)=\alpha J_{2}^{(\alpha+1)}\left(q^{2}, m^{2}\right),
$$

i.e. the diagram $I_{2}^{(\alpha)}\left(q^{2}, m^{2}\right)$ obeys the differential equation with the inhomogeneous term $J_{2}^{(\alpha+1)}\left(q^{2}, m^{2}\right)$ having very simple form: it contains only one-loop diagrams. To look it, we see that the last term in $J_{2}^{(\alpha)}\left(q^{2}, m^{2}\right)$ (see eq. (A4) ) is expressed through massive one loop $M_{\alpha_{1}, \alpha_{2}}\left(q^{2}, m^{2}\right)$ :

$$
M_{\alpha_{1}, \alpha_{2}}\left(q^{2}, m^{2}\right)=\int \frac{D k}{(q-k)^{2 \alpha_{1}}\left(k^{2}+m^{2}\right)^{\alpha_{2}}}=
$$

Indeed,

$$
S^{(1, \alpha)}\left(q^{2}, m^{2}\right)==A(1,1) M_{2-d / 2, \alpha}\left(q^{2}, m^{2}\right) .
$$

The one-loop diagram $M_{2-d / 2, \alpha}\left(q^{2}, m^{2}\right)$ can be evaluated by one of some effective methods, for example, by Feynman parameters.

We would like to note that $I_{2}^{(1)}\left(q^{2}, m^{2}\right)$ satisfies eq. (A10) with $\alpha=1$ that is not is of type of (35). But the integral $I_{2}^{(2)}\left(q^{2}, m^{2}\right)$ satisfies eq. (A10) with $\alpha=2$ that is of type of (35). So, it is convenient to rewrite (A9) with $I_{2}^{(2)}\left(q^{2}, m^{2}\right)$ in its r.h.s.:

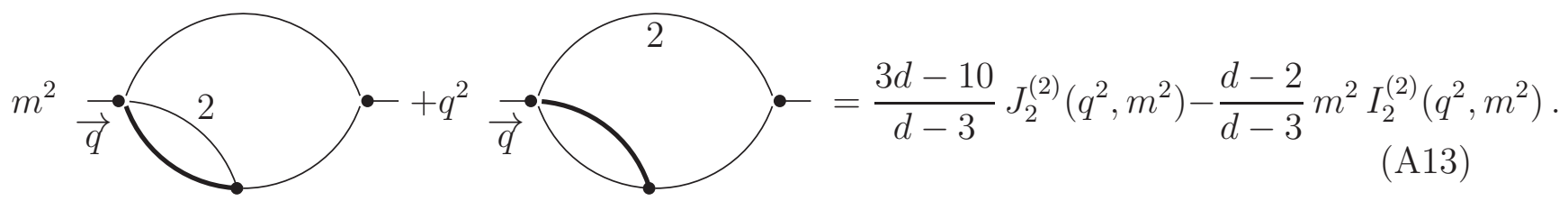

Now we should compare the IBP-based equations for $J_{2}^{(2)}\left(q^{2}, m^{2}\right)$ and $J_{2}^{(3)}\left(q^{2}, m^{2}\right)$ obtained in the right-hand sides of (A13) and (A10), respectively, with eq. (35). Since $J_{2}^{(3)}\left(q^{2}, m^{2}\right)=-\left(d / d m^{2}\right) J_{2}^{(2)}\left(q^{2}, m^{2}\right)$, consider only $J_{2}^{(2)}\left(q^{2}, m^{2}\right)$.

So, we should prepare the IBP-based equations for the massive one-loops $M_{\varepsilon, 2}\left(q^{2}, m^{2}\right)$ and $M_{\varepsilon, 3}\left(q^{2}, m^{2}\right)$. Applying IBP procedure with massive distinguished line to $M_{\varepsilon, 2}\left(q^{2}, m^{2}\right)$, we have

$$
(-3 \varepsilon) M_{\varepsilon, 2}\left(q^{2}, m^{2}\right)=\varepsilon\left[M_{1+\varepsilon, 1}\left(q^{2}, m^{2}\right)-\left(q^{2}+m^{2}\right) M_{1+\varepsilon, 2}\left(q^{2}, m^{2}\right)\right]-4 m^{2} M_{\varepsilon, 3}\left(q^{2}, m^{2}\right) .
$$

The corresponding applications of the IBP procedure with massless distinguished line to $M_{1+\varepsilon, 1}\left(q^{2}, m^{2}\right)$ and $M_{1+\varepsilon, 2}\left(q^{2}, m^{2}\right)$ lead to the following results:

$$
\begin{aligned}
& (1-4 \varepsilon) M_{1+\varepsilon, 1}\left(q^{2}, m^{2}\right)=M_{\varepsilon, 2}\left(q^{2}, m^{2}\right)-\left(q^{2}+m^{2}\right) M_{1+\varepsilon, 2}\left(q^{2}, m^{2}\right), \\
& -4 \varepsilon M_{1+\varepsilon, 2}\left(q^{2}, m^{2}\right)=2 M_{\varepsilon, 3}\left(q^{2}, m^{2}\right)-2\left(q^{2}+m^{2}\right) M_{1+\varepsilon, 3}\left(q^{2}, m^{2}\right),
\end{aligned}
$$

The last equations has the following form

$$
\left[-4 \varepsilon-\left(q^{2}+m^{2}\right) \frac{d}{d m^{2}}\right] M_{1+\varepsilon, 2}\left(q^{2}, m^{2}\right)=-\frac{d}{d m^{2}} M_{\varepsilon, 2}\left(q^{2}, m^{2}\right)
$$


Putting (A15) to (A14), we have after little algebra

$\left.-4 \varepsilon(1-3 \varepsilon) M_{\varepsilon, 2}\left(q^{2}, m^{2}\right)=-2 \varepsilon(1-4 \varepsilon)\left(q^{2}+m^{2}\right) M_{1+\varepsilon, 2}\left(q^{2}, m^{2}\right)\right]-4(1-4 \varepsilon) m^{2} M_{\varepsilon, 3}\left(q^{2}, m^{2}\right)$,

which transforms to

$$
\left[-4 \varepsilon(1-3 \varepsilon)-2(1-4 \varepsilon) \frac{d}{d m^{2}}\right] M_{\varepsilon, 2}\left(q^{2}, m^{2}\right)=-2 \varepsilon(1-4 \varepsilon)\left(q^{2}+m^{2}\right) M_{1+\varepsilon, 2}\left(q^{2}, m^{2}\right)
$$

So, eqs. (A17) and (A19) can be frustrated as a system of equations having a form similar to equation (35).

\section{References}

[1] K. G. Chetyrkin and F. V. Tkachov, Nucl. Phys. B 192 (1981) 159; F. V. Tkachov, Phys. Lett. B 100 (1981) 65; A. N. Vasiliev, Y. M. Pismak and Yu. R. Khonkonen, Theor. Math. Phys. 46 (1981) 104

[2] K. G. Chetyrkin et al., Nucl. Phys. B 174 (1980) 345; A. V. Kotikov, Phys. Lett. B 375 (1996) 240.

[3] A. V. Kotikov and S. Teber, Phys. Rev. D 89 (2014) no.6, 065038

[4] A. Grozin, In *Grozin, Andrey: Lectures on QED and QCD* 1-156 hep-ph/0508242];

A. G. Grozin, Int. J. Mod. Phys. A 27 (2012) 1230018

[5] S. Teber and A. V. Kotikov, Theor. Math. Phys. 190 (2017) no.3, 446; A. V. Kotikov and S. Teber, Phys. Part. Nucl. 50 (2019) no.1, 1

[6] A. I. Davydychev and J. B. Tausk, Phys. Rev. D 53 (1996) 7381

[7] D. J. Broadhurst, Z. Phys. C 47 (1990) 115.

[8] B. A. Kniehl, A. F. Pikelner and O. L. Veretin, JHEP 1708 (2017) 024; A. Pikelner, Comput. Phys. Commun. 224 (2018) 282

[9] L. H. Ryder, "Quantum Field Theory", Cambridge University Press, 1996.

[10] M. Kalmykov, V. Bytev, B. A. Kniehl, S. O. Moch, B. F. L. Ward and S. A. Yost, arXiv:2012.14492 [hep-th].

[11] T. Regge, Algebraic Topology Methods in the Theory of Feynman Rela- tivistic Amplitudes, Battelle Rencontres: 1967 Lectures in Mathematics and Physics, C.M. DeWitt, J.A. Wheeler (Eds.), (W.A. Benjamin, New York, 1968), pp. 433-458.

[12] Golubeva, V. A.: Some problems in the analytical theory of Feynman integrals. Russ. Math. Surv. 31139 (1976)

[13] A. V. Kotikov, Phys. Lett. B 254 (1991) 158

[14] E. Remiddi, Nuovo Cim. A 110 (1997) 1435. 
[15] A. V. Kotikov, Phys. Lett. B 259 (1991) 314

[16] T. Gehrmann and E. Remiddi, Nucl. Phys. B 580 (2000) 485

[17] A. V. Kotikov, Phys. Lett. B 267 (1991) 123; Mod. Phys. Lett. A 6 (1991) 3133

[18] Z. Bern, L. J. Dixon, D. C. Dunbar and D. A. Kosower, Nucl. Phys. B 425 (1994) 217

[19] A. V. Kotikov, Ukr. Fiz. Zh. (Russ. Ed. ) 37 (1992) 303.

[20] N. N. Bogoliubov and O. S. Parasiuk, Acta Math. 97 (1957) 227; K. Hepp, Commun. Math. Phys. 2 (1966) 301; W. Zimmermann, Commun. Math. Phys. 15 (1969) 208 [Lect. Notes Phys. 558 (2000) 217].

[21] N. N. Bogolyubov and D. V. Shirkov, Intersci. Monogr. Phys. Astron. 3 (1959) 1.

[22] D. I. Kazakov, Phys. Lett. 133B (1983) 406; Theor. Math. Phys. 58 (1984) 223; D. I. Kazakov, Theor. Math. Phys. 62 (1985) 84; N. I. Usyukina, Theor. Math. Phys. 54 (1983) 78; V. V. Belokurov and N. I. Usyukina, J. Phys. A 16 (1983) 2811; Theor. Math. Phys. 79 (1989) 385.

[23] D. I. Kazakov and A. V. Kotikov, Theor. Math. Phys. 73 (1988) 1264; A. V. Kotikov, Theor. Math. Phys. 78 (1989) 134.

[24] D. I. Kazakov and A. V. Kotikov, Nucl. Phys. B 307 (1988) 721 [Nucl. Phys. B 345 (1990) 299].

[25] A. V. Kotikov, Mod. Phys. Lett. A 6 (1991) 677

[26] A. V. Kotikov, Int. J. Mod. Phys. A 7 (1992) 1977.

[27] D. I. Kazakov and A. V. Kotikov, Phys. Lett. B 291 (1992) 171; Yad. Fiz. 46 (1987) 1767; D. I. Kazakov, A. V. Kotikov, G. Parente, O. A. Sampayo and J. Sanchez Guillen, Phys. Rev. Lett. 65 (1990) 1535

[28] F. Herzog, S. Moch, B. Ruijl, T. Ueda, J. A. M. Vermaseren and A. Vogt, Phys. Lett. B 790 (2019) 436

[29] J. Blumlein, S. Klein and B. Todtli, Phys. Rev. D 80 (2009) 094010

[30] A. Behring, J. Blumlein, A. De Freitas, A. von Manteuffel, K. Schönwald and C. Schneider, arXiv:2101.05733 [hep-ph].

[31] J. Ablinger, J. Blumlein, A. De Freitas, A. Goedicke, M. Saragnese, C. Schneider and K. Schönwald, Nucl. Phys. B 955 (2020) 115059

[32] J. Blumlein and C. Schneider, Int. J. Mod. Phys. A 33 (2018) no.17, 1830015

[33] J. Fleischer et al., Phys. Lett. B 462 (1999) 169.

[34] J. Fleischer et al., Phys. Lett. B 417 (1998) 163 
[35] J. Fleischer et al., Nucl. Phys. B 547 (1999) 343; Acta Phys. Polon. B 29 (1998) 2611.

[36] A. V. Kotikov, Particles 3 (2020) no.2, 394

[37] B. A. Kniehl et al., Nucl. Phys. B 738 (2006) 306; Nucl. Phys. B 948 (2019) 114780

[38] B. A. Kniehl and A. V. Kotikov, Phys. Lett. B 638 (2006) 531; Phys. Lett. B 712 (2012) 233.

[39] J. A. M. Vermaseren, Int. J. Mod. Phys. A 14 (1999) 2037; J. Blumlein and S. Kurth, Phys. Rev. D 60 (1999) 014018

[40] A. V. Kotikov, Phys. Atom. Nucl. 57 (1994) 133; A. V. Kotikov and V. N. Velizhanin, hep-ph/0501274.

[41] A. Devoto and D. W. Duke, Riv. Nuovo Cim. 7N6 (1984) 1.

[42] B. A. Kniehl et al., Phys. Rev. Lett. 97 (2006) 042001; Phys. Rev. D 79 (2009) 114032;

Phys. Rev. Lett. 101 (2008) 193401; Phys. Rev. A 80 (2009) 052501;

[43] A. V. Kotikov and L. N. Lipatov, Nucl. Phys. B 661 (2003) 19; in: Proc. of the XXXV Winter School, Repino, S'Peterburg, 2001 (hep-ph/0112346).

[44] L. Bianchi et al., Phys. Lett. B 725 (2013) 394

[45] A. V. Kotikov and L. N. Lipatov, Nucl. Phys. B582 (2000) 19.

[46] L. N. Lipatov, Sov. J. Nucl. Phys. 23 (1976) 338; V. S. Fadin et al., Phys. Lett. B 60 (1975) 50; E. A. Kuraev, et al., Sov. Phys. JETP 44 (1976) 443; Sov. Phys. JETP 45 (1977) 199; I. I. Balitsky and L. N. Lipatov, Sov. J. Nucl. Phys. 28 (1978) 822; JETP Lett. 30 (1979) 355.

[47] A. V. Kotikov et al., Phys. Lett. B 557 (2003) 114.

[48] A. V. Kotikov et al., Phys. Lett. B 595 (2004) 521.

[49] A.V. Kotikov et al., J. Stat. Mech. 0710 (2007) P10003; Z. Bajnok, R.A. Janik, and T. Lukowski, Nucl. Phys. B 816 (2009) 376.

[50] T. Lukowski et al., Nucl. Phys. B 831, 105 (2010).

[51] C. Marboe et al., JHEP 1507 (2015) 084

[52] C. Marboe and V. Velizhanin, JHEP 1611 (2016) 013

[53] M. Staudacher, JHEP 0505 (2005) 054; N. Beisert and M. Staudacher, Nucl. Phys. B 727 (2005) 1.

[54] E. Remiddi and J. A. M. Vermaseren, Int. J. Mod. Phys. A 15 (2000) 725

[55] A. I. Davydychev and M. Y. Kalmykov, Nucl. Phys. B 699 (2004) 3. 
[56] A. V. Kotikov, In *Diakonov, D. (ed.): Subtleties in quantum field theory* 150-174 arXiv:1005.5029 [hep-th]]; Phys. Part. Nucl. 44 (2013) 374; A. V. Kotikov and A. I. Onishchenko, arXiv:1908.05113 [hep-th].

[57] A. V. Kotikov, Theor. Math. Phys. 176 (2013) 913; Theor. Math. Phys. 190 (2017) no.3, 391

[58] T. Gehrmann et al., JHEP 1203 (2012) 101.

[59] J. M. Henn, Phys. Rev. Lett. 110 (2013) 251601; J. Phys. A 48 (2015) 153001

[60] L. Adams and S. Weinzierl, Phys. Lett. B 781 (2018) 270

[61] L. Adams, C. Bogner, A. Schweitzer and S. Weinzierl, J. Math. Phys. 57 (2016) no.12, 122302

[62] C. G. Papadopoulos, JHEP 1407 (2014) 088

[63] S. Weinzierl, arXiv:1912.02578 [hep-ph]; arXiv:2012.08429 [hep-th].

[64] M. Argeri and P. Mastrolia, Int. J. Mod. Phys. A 22 (2007) 4375

[65] R. N. Lee, JHEP 1504 (2015) 108; JHEP 1810 (2018) 176; R. N. Lee and A. I. Onishchenko, JHEP 1912 (2019) 084;

[66] R. N. Lee and A. A. Pomeransky, arXiv:1707.07856 [hep-th].

[67] A. B. Goncharov, math/0103059 [math.AG].

[68] C. Duhr, arXiv:1411.7538 [hep-ph].

[69] A. Kotikov et al., Nucl. Phys. B 788 (2008) 47.

[70] U. Aglietti and R. Bonciani, Nucl. Phys. B 668 (2003) 3; U. Aglietti, R. Bonciani, G. Degrassi and A. Vicini, Phys. Lett. B 595 (2004) 432; Phys. Lett. B 600 (2004) 57; JHEP 0701 (2007) 021

[71] J. Henn, A. V. Smirnov, V. A. Smirnov, M. Steinhauser and R. N. Lee, JHEP 1703 (2017) 139; J. Ablinger, J. Blumlein, P. Marquard, N. Rana and C. Schneider, Phys. Lett. B 782 (2018) 528; J. Ablinger, J. Blumlein, P. Marquard, N. Rana and C. Schneider, Nucl. Phys. B 939 (2019) 253

[72] J. Ablinger, J. Blumlein and C. Schneider, J. Math. Phys. 52 (2011) 102301

[73] D. D. Canko, C. G. Papadopoulos and N. Syrrakos, JHEP 2101 (2021) 199; D. D. Canko and N. Syrrakos, arXiv:2010.06947 [hep-ph]; N. Syrrakos, arXiv:2012.10635 [hep-ph].

[74] R. N. Lee, A. V. Smirnov and V. A. Smirnov, JHEP 1803 (2018) 008

[75] R. N. Lee, arXiv:2012.00279 [hep-ph].

[76] B. Kol, arXiv:1507.01359 [hep-th]. 
[77] B. Kol, A. Schiller and R. Shir, JHEP 2101 (2021) 165

[78] P. Vanhove, arXiv:1807.11466 [hep-th]

[79] J. Broedel, C. Duhr, F. Dulat, B. Penante and L. Tancredi, arXiv:1807.00842 [hep-th];

J. Broedel and A. Kaderli, J. Phys. A 53 (2020) no.24, 245201

[80] M. Walden and S. Weinzierl, arXiv:2010.05271 [hep-ph]; L. Adams, E. Chaubey and S. Weinzierl, PoS LL 2018 (2018) 069 [arXiv:1807.03599 [hep-ph]].

[81] J. Campert, F. Moriello and A. Kotikov, arXiv:2011.01904 [hep-ph].

[82] J. Broedel, C. Duhr, F. Dulat and L. Tancredi, JHEP 1805 (2018) 093

[83] M. Y. Kalmykov and B. A. Kniehl, Nucl. Phys. B 809 (2009) 365

[84] M. A. Bezuglov, A. I. Onishchenko and O. L. Veretin, Nucl. Phys. B 963 (2021) 115302 